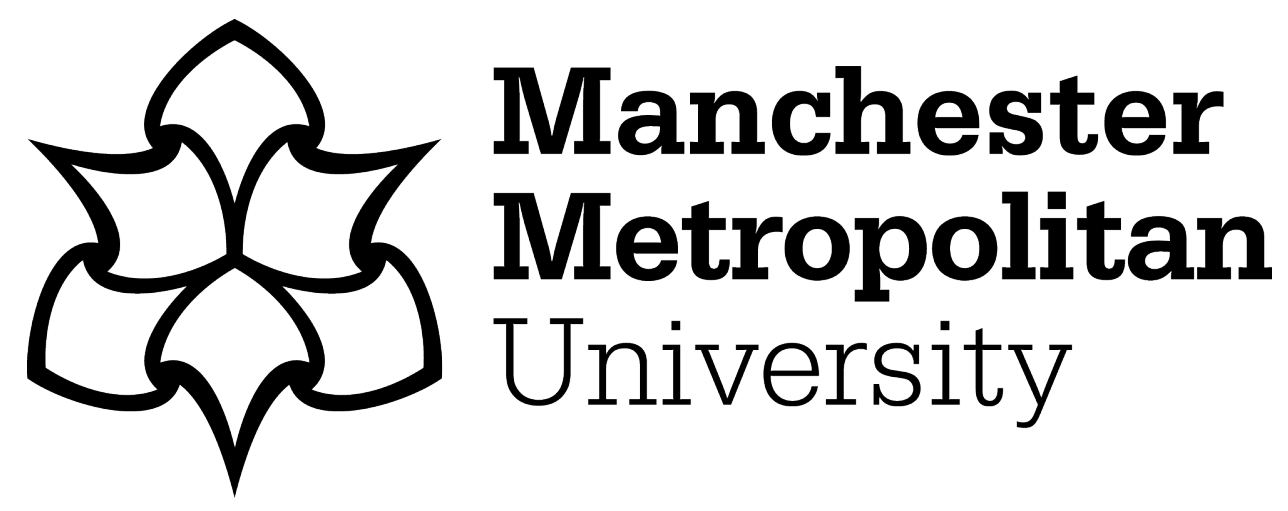

Aguiar Noury, G, Tsekeni, M, Morales, V, Burke, R, Palomino, M and Masala, GL (2021) Experiment protocol for human-robot interaction studies with seniors with mild cognitive impairments. In: International KES Conference on Human Centred Intelligent Systems 2020 (KES HCIS 2020), 17 June 2020 19 June 2020, Virtual.

Downloaded from: https://e-space.mmu.ac.uk/626170/

Version: Accepted Version

Publisher: Springer

DOI: https://doi.org/10.1007/978-981-15-5784-2_20

Please cite the published version 


\title{
Experiment protocol for human-robot interaction studies with seniors with mild cognitive impairments
}

\author{
Gabriel Aguiar Noury ${ }^{1}$, Margarita Tsekeni ${ }^{1}$, Vanessa Morales ${ }^{1}$, Ricky Burke ${ }^{1}$, \\ Marco Palomino ${ }^{1}$, Giovanni L. Masala ${ }^{2}$. \\ ${ }^{1}$ University of Plymouth, Plymouth, United Kingdom \\ ${ }^{2}$ Manchester Metropolitan University, Manchester, United Kingdom
}

\begin{abstract}
While assistive robotics (AR) have shown promise in supporting seniors with daily life activities and psycho-social development, evaluation of AR systems present novel challenges. From a technical point of view, reproducing HRI experiments has been problematic due to the lack of protocols, standardization, and benchmarking tools, which ultimately impairs the evaluation of previous experiments. On the other hand, working with seniors with cognitive decline presents a major design challenge for researchers, since communication skills, state of mind and attention of participants is compromised. To address these challenges, this paper presents practical recommendations and a protocol for conducting HRI experiments with seniors with mid cognitive decline (MCI).
\end{abstract}

Keywords: Assistive technology, human-robot interaction, measurement and evaluation, experimental design.

\section{Introduction}

The worldwide elderly population is expected to reach approximately one billion in 2030 and 1.5 billion in 2050 [1]. This global population ageing phenomenon is increasing the burden of healthcare systems, who are looking for innovative solutions to satisfy this new demand while maintaining the quality and affordance of care delivery.

One area of technology that shows promise in solving these challenges is the assistive robotics (AR) [2]. AR assumes the primary role of providing help to carers or directly to patients. From automating physical tasks that a senior can no longer do to encouraging social behaviour, AR is a growing area of research with potential benefit for eldercare.

The advance of effective methods and tools to evaluate human-robot interactions (HRIs) with seniors with cognitive decline is lacking. We have adopted and modified methods of testing and evaluating robots from the field of human-computer interaction, but HRI is not identical [3]. Most importantly, the design methodology of HRI research studies that produce verifiable, reliable, and reproducible results has been a major challenge in the last decade [4]. Among other reasons, this is because the experiment procedures have not been standardized [5]. 
On the other hand, conducting studies upon patients with cognitive impairment represents always a challenge, even more, if considering psychological factors as state of mind, concentration and technology dexterity (i.e., [6, 7]). Cognitive impairment is a common problem within the elderly population with an occurrence rate of approximately 21.5 to 71.3 per 1,000 person-years in seniors [8]. The elderly population with cognitive impairments finds it difficult to distinguish and differentiate between simultaneous sensory stimulations and become confused easily. They also develop communication disorders which difficult their ability to express their views. Besides, ageing can reduce the ability to see, hear, and touch. All of these represent a challenge for researchers to gather useful and unbiased data.

This highlights the need for developing common protocols as an open research issue in HRI with seniors with cognitive decline. Therefore, this paper has the objective of proposing a protocol for evaluating HRI with seniors with mild cognitive decline (MCI). This study is for researchers working on live interactions, interaction with products of reduced functionality, mock-ups operated in Wizard-of-Oz mode and acted demo [9].

In section 2, we describe the methodology used in this study. Then, section 3 explores the design of the University of Plymouth's Robot Home; a lab for the evaluation of HRI. Next, section 4 presents practical guidelines for selecting senior participants with MCI. Section 5 explores the different techniques for collecting data during HRI experiments. Finally, section 6 proposes a protocol for conducting HRI experiments with seniors with MCI. Conclusions and further research are presented in section 7.

\section{Methodology}

This paper builds upon the lessons learned from setting up the University of Plymouth Robot Home lab for senior participants with cognitive decline, and the twenty-two dropout sessions made during the EHealth Productivity and Innovation in Cornwall and the Isles of Scilly EPIC project [10]. The lab resembles a living room that follows care environmental guidelines adopted from a desk research and expert consultation with occupational therapists.

The study does not focus on patients with severe dementia, hearing or visual impairments - this includes patients that suffer from hallucinations or low consciousness level. Instead, this paper gives recommendations for working with participants with MCI; "stage between the expected cognitive decline of normal ageing and the more serious decline of dementia" [11]. It can involve deterioration of memory, attention, and cognitive function that are greater than expected based on age and educational level. These subjects are becoming the focus of many studies and early intervention trials since MCI is about four-times greater than dementia [12]. Moreover, this paper does not discuss ethical concerns since they have been deeply covered by previous studies [13]. 


\section{An example of robotics lab for HRI: The Robot Home}

The Robot Home set up started in May 2019 and finished in September 2019. It was funded by the Interreg 2 Seas Mers Zeeën Ageing Independently (AGE'In) project. The aim of the lab design was to create a facility that follows strict care environmental guidelines for the evaluation of an HRI with seniors and vulnerable participants. A lab that will allow researchers to evaluate the acceptability and usability of AR technologies while supporting the integration of third party devices for the simulation of different scenarios related to smart homes and independent living. Figure 1 shows the lab that resembles a living room; a relatable, but a secure place that will reduce cognitive bias from experiment participants.

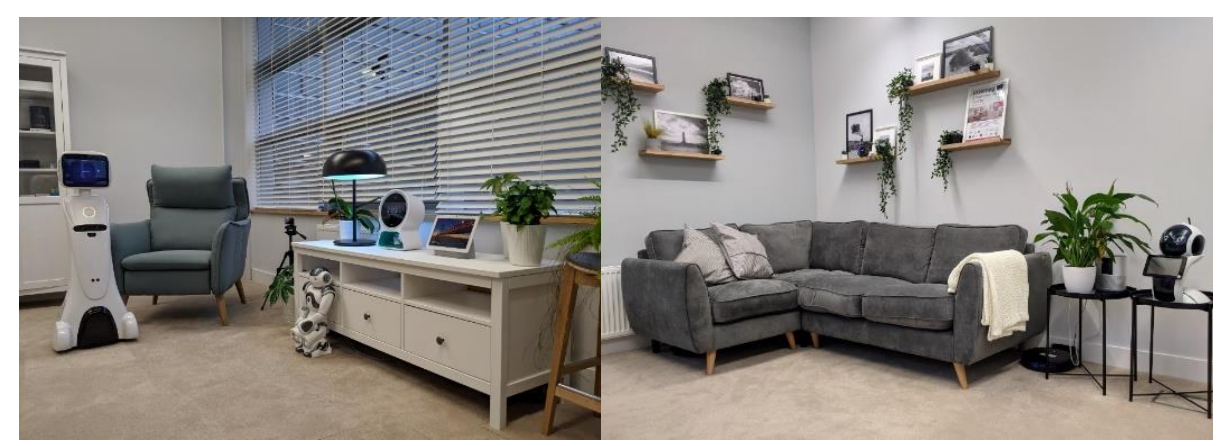

Fig. 1. Robot Home, University of Plymouth. From left to right, the robots in the picture are; AMY A1, NAO, and QBo One.

The project counted with the support of two interior designers, one architect, one multimedia engineer, and three occupational therapists from the University of Plymouth.

The work was divided into four main activities;

- Market Study; to identify AR, sensors and IoT devices for the evaluation and enhancement of HRI.

- Multimedia Study; to identify the cameras, microphones and accessories needed, and the location of the same in the room.

- Care Environment Design; to generate and evaluate different concepts for the design of the lab.

- Implementation; including room adaptation, and setting up the equipment.

The interior design team generated more than 25 different concepts. The most promising solutions were then evaluated by the architect and occupational therapist, and the design was refined through their feedback. The sofas of the room follow regulations on the seat height and depth, and arm height to allow participants to sit and stand up without difficulties. In the same way, the colour pattern of the room was chosen to generate a calm environment, but also to reduce light reflection and colour interference while tracking people's faces. 
The lab's carpet is a non-slippery carpet, soft enough to provide some protection against injury from falls to people and robots, but not to interfere with the mobility of the systems. Finally, the blinds were placed to control the amount of natural light that enters the room and to hide the cameras to be placed behind.

For the data collection, the room counts with four GoPro Hero 7 cameras and four modify GoPro with different lenses (Table 1). The cameras allow us to capture 1440 resolution and 60 frames per second, with a 4:3 aspect ratio and wide field of view. The integrated application of the cameras allows us to control and monitor several cameras in real-time. The position of the cameras could be adjusted depending on the interaction setting, and they will support further studies.

To analyse participants behaviour, the room has four Kinect Azure Cameras, for building computer vision and speech models (Table 1). The cameras will show instance segmentation, 2D key points, and 3D joints. This provides a fully articulated body tracking of multiple participants. Besides, through Azure cognitive services, researchers will be able to detect and identify peoples' emotions during the experiments. Table 1 presents a list of the sensors used in the lab.

In term of robotic platforms, the room counts with a NAO robot (commonly used as an example of socially assistive robots [14]), the AMY A1 telepresence robot (a commercially available telepresence robot used to explore how RAS could address social isolation issues [15]), and the Qbo One robot (a research platform used for its potential as a robot companion at home [16]). These robotic platforms will allow researchers to conduct different studies.

For the integration of smart devices, the room counts with both Google Assistant and Alexa hub. This is complemented with two smartphones; Pixel 3a and iPhone $\mathrm{XR}$, that allow researchers to evaluated AR technologies that work with mobile phones. Wearable devices such as the Apple Watch Series 4 and the Samsung Galaxy Watch allow researchers to monitor participants resting heart rate (while the user is not performing a physical activity), as a channel for gathering psychophysiological measurements.

Table 1. Robot Home sensors

\begin{tabular}{ccc} 
Sensor & Item & Description \\
\hline Camera & GoPro Hero 7 & 1440 resolution, 60 fps, 4:3 ratio \\
Body Tracker & Azure Kinect & 2D and 3D joint extraction \\
Microphone & Azure Kinect & On-board microphone array \\
Facial/Emotion recognition & Azure Kinect & Azure cognitive services \\
Heart rate & Apple Watch 4 & ECG monitor \\
Room temperature & Microbot Alert & Temperature, humidity, air pres- \\
& & sure, light intensity, noise level
\end{tabular}

The room also counts with four smart switches to control the heating and air conditioning of the room to be used in home automation scenarios. The smart switches can also control indoor weather, light, and noise sensors for further applications. 


\section{$4 \quad$ Guidelines for participant selection}

It is important that researchers report the cognitive level of their experiment participants. However, identifying the cognitively level of seniors is a difficult task [17]. In the UK, a general practitioner, or a specialist at a memory clinic or hospital, can only diagnose MCI [18]. Therefore, relied first on the assessment records.

If not available, there are tools to assess the cognitive impairment of seniors as the General Practitioner Assessment of Cognition [19], or the Mini-Cog test [20]. They are short validated tests that a researcher can use. Running these assessments provides a baseline for homogenous samples.

- We recommend using the Montreal Cognitive Assessment (a screening tool broadly use for detecting MCI [21]). It takes around ten minutes to administer and it also assesses the attention and verbal fluency of the senior.

In the same way, it is important to measure the participants hearing and visual impairments since this will influence the HRI.

- We recommend using the Hearing Handicap Inventory for the Elderly Screening Version [22] and the Amsler grid for assessing visual loss [23], which are tests that are easy to conduct and assess.

\section{$5 \quad$ Guidelines for data collection}

Traditionally in HRI studies, there are three main methods to collect participants feedback: self-report, behavioural, and psychophysiological measures [4].

\subsection{Self-report measures}

Self-report measures are one of the most used methods in HRI. With questionnaires, researchers explore views of participants regarding the appearance, interaction, and overall satisfaction of the robot. These measures are easy to gather and analyse, involving simple statistical techniques.

- While working with self-report questionnaires with seniors with MCI, researchers should take into consideration the time between the interaction and the assessment. We have seen that between 15 to 30 minutes after the interaction, some seniors tend to forget sensible elements.

- MCI affects the communication skills of the participants, who are no longer able to describe, in-depth, their feelings, attitudes and recommendations towards the technology being assessed.

Questionnaires must be carefully designed and tested before beginning an experiment. The next clinical questionnaires constitute a useful source for researchers:

- ICECAP-O [24] or the WEMWBS [25]: To assess senior general wellbeing. 
- CES-D [26] or Giervald scale [27]: To explore depression and loneliness of seniors.

- Duke Social Index or the Lubben Social Scale [27]: To assess social isolation.

- SF-36 questionnaire [28]: To study HRI impact in senior general health.

It is useful for HRI researchers to explore these tools since the wording and question structure allow them to frame their assessments under validated questionnaires' protocols.

\subsection{Behavioural measures}

These measures focus on the conduct, functioning and actions performed by the participants during experiments.

The data is gathered through video recording or researchers' observations. For instance, valence and arousal, the time spent looking at the robot, the time mutually looking at a specific cue, or the time spent in open interaction with the robot. The analysis of the data frequently requires independent coders. For instance, [29] or [30] are some of the multiple examples of HRI that gather behavioural measures.

Non-verbal communication is essential for evaluations. As ageing progresses, body language and physical contact become the main communication channel.

- Gestures, facial expression and body language can be recorded and the video analysed by different coders.

- We recommend using an open coding system by independent researchers, using a five-point Likert scale assessing valence and arousal.

Arousal and valence scales can label quality and intensity of affective body language by utilising a large range of affective states [31], disregarding if the participants are standing or seated. These scales are effective in describing a persons' affective behaviours during social interactions. Finally, valence and arousal had better characterise experimental and clinical findings than a categorical emotional [32].

\subsection{Psychophysiology measures}

Psychophysiology measures focus on the interaction between the mind and body [33]. The most common measures used in controlled HRI experiments are electroencephalography, heart rate variability, skin conductance response, interbeat interval, blood pressure, respiratory sinus arrhythmia and electromyography.

- The use of psychophysiological measures is challenging while working with seniors. For instance, while using electromyography techniques, locating the electrode placement, and making sure that the appropriate amounts of conducting gel or paste are being used, is a difficult task with seniors.

While there are some devices that offer alternatives to the use of traditional EEG (i.e.; Emotiv EPOC+), these intrusive devices will only interfere with the results of the evaluation. 
- We do recommend the use of smartwatches capable of reading resting blood pressure. Seniors have used watches before; thus, the technology will not overwhelm them.

\section{Experiment protocol}

Performing short-term pilots in the field of HRI has a major shortcoming: participants are every so often excited for interacting with a robot for the first time (this is the novelty effect). Besides, due to the loneliness that residents experience at care homes, seniors are eager to interact with researchers and provide positive feedback [13].

On the other hand, uncertainty, drastic changes on daily routine or loss of control affect deeply senior with MCI. To address these issues, we propose a protocol focussed on four pillars (Figure 2).

\begin{tabular}{|c|c|c|c|}
\hline Initial Work & Experiment Setup & Robot Introduction & Assessment \\
\hline \multirow{2}{*}{$\begin{array}{l}\text { Co-planning of the } \\
\text { experiment with } \\
\text { carers }\end{array}$} & \multirow{6}{*}{\begin{tabular}{|l|} 
Setup outside the \\
experiment room
\end{tabular}} & \multirow{3}{*}{\begin{tabular}{|l|}
$\begin{array}{l}\text { First contact of research } \\
\text { participants with the robot }\end{array}$ \\
Let the participants interact \\
freely with the robot
\end{tabular}} & \multirow{3}{*}{\begin{tabular}{|l} 
Run the designed \\
experiment \\
$\begin{array}{l}\text { Concluded the interaction } \\
\text { start the self-reported } \\
\text { tool }\end{array}$ \\
\end{tabular}} \\
\hline & & & \\
\hline \multirow{4}{*}{$\begin{array}{l}\text { Visit and meet the } \\
\text { participants before } \\
\text { the intervention }\end{array}$} & & & \\
\hline & & $\begin{array}{l}\text { Do not run any pre- } \\
\text { program app or skill. }\end{array}$ & Listen and work through \\
\hline & & \multirow{2}{*}{$\begin{array}{c}\text { Take the robot out of the } \\
\text { room if participants are } \\
\text { upset }\end{array}$} & \\
\hline & & & $\begin{array}{l}\text { Rephrase questions if } \\
\text { needed }\end{array}$ \\
\hline
\end{tabular}

Fig. 2. Summary of the experiment protocol.

During the initial work;

- First, contact the healthcare organization or family member regarding the study ethics concerns.

- Then, schedule with the seniors' caregivers an appropriate time for the experiment. During certain times seniors are more lucid or in a good mood.

- One week before the experiment, request the caregivers to talk with the seniors about the experiment, about the robot that is visiting them and the day and hour when this will take place. If possible, ask the carers to show pictures or videos of the robot.

- Visit the site before the evaluation. Choose the room where the study will take place, select the research participants, and have a first interaction with them.

The day of the experiment, we recommend reducing contact with the participants while setting up. The day of the experiment;

- Do the initial setup of the robot outside the experiment room.

- Once ready, let the participant or participants enter the experiment room and sit down or stand up according to the experiment design.

- Researchers should introduce themselves and explain the activity to the senior. 
- Once the senior feels at ease, start the recording equipment to be used in the research.

At this point, the robot will be ready to enter the experiment room:

- Position it where the person can see it as clearly as possible. If the senior is sitting down, we recommend having the robot at the same level.

- Let the subject interact freely with the robot, allowing the participant to become familiarized with the device while recording any feedback.

- During this initial interaction, allow the carers to be in the experiment room.

- Make sure that no application is running, except for those which are the focus of the experiment (i.e.; pre-program apps from the manufactures).

- If the participant gets upset or distressed to the point where the experiment cannot begin; take the robot out of the room, allow carers to calm down the participants, and with their approval, repeat the robot introduction.

This initial interaction could take between one to five minutes, depending upon senior engagement. If the senior level of consciousness is low, it is unlikely that the senior will react to the technology. Once this initial interaction has been completed;

- Run the designed experiment.

- The experiment time with each senior may vary, and rushing the senior will influence the evaluation.

- Immediately after concluding the interaction, with the robot still in the room, proceed with any self-reported method chosen for the collection of data.

- Prompt seniors to elaborate their answers by asking open-ended questions.

- Listen patiently and work through to deeper questions of the evaluation. If the senior gets confused, or upset by the question, or by their communication skills, change the subject. The researcher can rephrase the question and ask it again later on.

- The researcher should build upon the participants' answers in order to avoid the senior feeling she/he is being interrogated.

Finally, in long term pilots, it has been reported that once the experiment has concluded, participants feel depressed due to the departure of the robot or the researchers. It is unethical therefore for the researcher to overlook this effect of the intervention. We strongly recommend for pilots that take more than two weeks to debrief seniors during the last day of the intervention that researchers and robotic platform will leave.

\section{Conclusion}

The aim of the paper was to guide HRI researchers while conducting experiments with seniors with MCI. Besides contributing to researchers without prior training in clinical sciences, we raise awareness about the importance of experiment standardization.

Conducting experiments with seniors with MCI is challenging. Communication deterioration, poor eyesight and hearing difficulties make the evaluation difficult. Sen- 
iors get easily irritated with voice recognition technologies and new technologies can frighten them. On the other hand, evaluating how a senior reacts to and is affected by $\mathrm{AR}$, is a methodological conundrum. The senior could be happy due to the novelty effect of the robot, or because of the interaction with people. Still, seniors could also be upset due to the change in their daily routine, or confused due to the presence of a robot. Researchers should follow guidelines to mitigate these effects.

Working with care questionnaires to assess the cognition of our participants, hearing and visual impairments, allow readers to understand and recreate our experiments. Clinical tests also allow researchers to understand the wording and framing of questions to support our evaluations.

Finally, the HRI community needs to establish methods for AR first contact with seniors to ensure the integrity of the data collected and the seniors' wellbeing. It is unethical for the researchers to focus only on the technology, and not in the interaction that they are directly having on the experiment participants. Every time a researcher enters a care establishment, they should follow a conduct protocol.

To address these issues, this research has established recommendations presented as practical steps for researchers to follow. This is by no means an exhaustive list and will evolve with the state-of-the-art and the new opportunities that AR will bring.

\section{Acknowledgement}

Authors of this paper acknowledge the funding provided by the Interreg 2 Seas Mers Zeeën AGE'In project (2S05-014) to support the work in the research described in this publication.

\section{References}

1. Lee, Y., Hwang, J., Lim, S., Kim, J.T.: Identifying Characteristics of Design Guidelines for Elderly Care Environments from the Holistic Health Perspective. Indoor Built Environ. 22, 242-259 (2013).

2. UK-RAS: Robotics in Social Care: A Connected Care EcoSystem for Independent Living. (2017).

3. Kidd, C., Breazeal, C.: Human-robot interaction experiments: Lessons learned. In: Proceeding of AISB'05 Convention (2005).

4. Bethel, C.L., Burke, J.L., Murphy, R.R., Salomon, K.: Psychophysiological experimental design for use in human-robot interaction studies. In: Proceedings of the 2007 International Symposium on Collaborative Technologies and Systems, CTS. pp. 99-105 (2007).

5. EURobotics: Strategic Research Agenda For Robotics in Europe 2014-2020. IEEE Robot. Autom. Mag. 24, 171 (2014).

6. Desideri, L., Ottaviani, C., Malavasi, M., ... R. di M.-C. in H., 2019, undefined: Emotional processes in human-robot interaction during brief cognitive testing. Elsevier.

7. Di Nuovo, A., Varrasi, S., Lucas, A., Conti, D., McNamara, J., Soranzo, A.: Assessment of Cognitive skills via Human-robot Interaction and Cloud Computing. J. Bionic Eng. 16, 526-539 (2019).

8. Tricco, A.C., Soobiah, C., Lillie, E., Perrier, L., Chen, M.H., Hemmelgarn, B., Majumdar, S.R., Straus, S.E.: Use of cognitive enhancers for mild cognitive impairment: protocol for a systematic review and network meta-analysis. Syst. Rev. 1, 25 (2012).

9. Xu, Q., Ng, J., Tan, O., Huang, Z., Tay, B., Park, T.: Methodological Issues in Scenario- 
Based Evaluation of Human-Robot Interaction. Int. J. Soc. Robot. 7, 279-291 (2015).

10. Jones, R., Asthana, S., Walmsley, A., Sheaff, R., Milligan, J., Paisey, M., Aguiar Noury, G.: Developing the eHealth sector in Cornwall. , Plymouth (2019).

11. Petersen, R.C., Smith, G.E., Waring, S.C., Ivnik, R.J., Tangalos, E.G., Kokmen, E.: Mild Cognitive Impairment. Arch. Neurol. 56, 303 (1999).

12. Eshkoor, S.A., Hamid, T.A., Mun, C.Y., Ng, C.K.: Mild cognitive impairment and its management in older people. Clin. Interv. Aging. 10, 687-93 (2015).

13. Berghmans, R.L.P., Meulen, R.H.J.T.: Ethical issues in research with dementia patients. Int. J. Geriatr. Psychiatry. 10, 647-651 (1995).

14. Comito, C., Caniot, M., Lagrue, E., Coignard, P., Fattal, C.: Psychological and symbolic determinants relating to the first meeting with a humanoid robot. Ann. Phys. Rehabil. Med. 59, e87 (2016).

15. Robot Center: Amy A1 Robot - Collaborative Robotics, https://www.robotcenter.co.uk/products/amy-a1-robot, last accessed 2020/02/11

16. Corpora: An Interactive Open Source Robot for Kids, Developers and Eldercare, http://thecorpora.com/, last accessed 2020/02/11.

17. Alzheimer's Society: Assessing cognition in older people : a practical toolkit for health professionals. 1-2 (2016).

18. NHS Choices: Tests for diagnosing dementia - Dementia guide - NHS Choices, https://www.nhs.uk/conditions/dementia/diagnosis-tests/, last accessed 2020/01/24.

19. Dementia Collaborative Research Centre-Assessment and Better Care: GPCOG | Home, http://gpcog.com.au/, last accessed 2020/01/24.

20. Hartford Institute for Geriatric Nursing: Mental Status Assessment of Older Adults: The Mini-Cog. Alzheimer's Dement. 13, 325-373 (2017).

21. MoCA: MOCA Montreal Cognitive Assessment. 2004 (2004).

22. Servidoni, A.B., Conterno, L. de O.: Hearing Loss in the Elderly: Is the Hearing Handicap Inventory for the Elderly - Screening Version Effective in Diagnosis When Compared to the Audiometric Test? Int. Arch. Otorhinolaryngol. 22, 1-8 (2018).

23. Schuchard, R.A.: Validity and Interpretation of Amsler Grid Reports. Arch. Ophthalmol. 111, 776-780 (1993).

24. Makai, P., Brouwer, W.B.F., Koopmanschap, M.A., Nieboer, A.P.: Capabilities and quality of life in Dutch psycho-geriatric nursing homes: an exploratory study using a proxy version of the ICECAP-O. Qual. Life Res. 21, 801-812 (2012).

25. Tennant, R., Hiller, L., Fishwick, R., Platt, S., Joseph, S., Weich, S., Parkinson, J., Secker, J., Stewart-Brown, S.: The Warwick-Edinburgh Mental Well-being Scale (WEMWBS): development and UK validation. Health Qual. Life Outcomes. 5, 63 (2007).

26. Radloff, L.S.: The use of the Center for Epidemiologic Studies Depression Scale in adolescents and young adults. J. Youth Adolesc. 20, 149-166 (1991)

27. de Jong Gierveld, J., van Tilburg, T.G.: Social Isolation and Loneliness. In: Encyclopedia of Mental Health: Second Edition. pp. 175-178 (2016).

28. Ware, J.E., Sherbourne, C.D.: The MOS 36-item short-form health survey (Sf-36): I. conceptual framework and item selection. Med. Care. 30, 473-483 (1992).

29. Sidner, C.L., Kidd, C.D., Lee, C., Lesh, N.: Where to look: a study of human-robot engagement. Proc. 9th Int. Conf. Intell. user interface - IUI '04. 78-84 (2004).

30. Breazeal, C., Kidd, C.D., Thomaz, A.L., Hoffman, G., Berlin, M.: Effects of nonverbal communication on efficiency and robustness in human-robot teamwork. In: 2005 IEEE/RSJ International Conference on Intelligent Robots and Systems, IROS. pp. 383-388 (2005).

31. Kapoor, A., Burleson, W., Picard, R.W.: Automatic prediction of frustration. Int. J. Hum. Comput. Stud. 65, 724-736 (2007).

32. Posner, J., Russell, J.A., Peterson, B.S.: The circumplex model of affect: An integrative approach to affective neuroscience, cognitive development, and psychopathology. Dev. Psychopathol. 17, 715-734 (2005).

33. Stern, R.M., Ray, W.J., Quigley, K.S.: Psychophysiological Recording. (2012). 